\title{
Identification of Vibrating Structures under Stochastic Loading Conditions
}

\author{
Taher G. Abu-El-Yazied ${ }^{1}$, Osama A. Montasser ${ }^{2}$, F. S. Al-Fares ${ }^{3}$ \\ ${ }^{I}$ Design and Production, Faculty of Engineering/ Ain Shams University, Egypt \\ ${ }^{2}$ Mechanical Power Engineering, Faculty of Engineering/Ain Shams University, Egypt, On leave to join the \\ British University in Egypt, BUE \\ ${ }^{3}$ College of Technological Studies, PAAET, Kuwait
}

\begin{abstract}
A stochastic identification technique is proposed to estimate both the order and parameters of multiinputs multi-outputs vibrating structural systems. A time-series model of an over-specified order is considered. The extraneous modes associated with the over-specified order model are distinguished from system modes by applying a backwards approach. The poles of the extraneous modes have been shown to be canceled by the extra zeroes. These poles are found to be generally inside the unit circle. Simulation examples have been presented to illustrate the effectiveness of the present proposed identification approach. Experimental runs on the vibration of a flexible truss, representing the tower of an overhead tower cranes, is carried out, the data of which was used to identify the system order and parameters.
\end{abstract}

Keywords: Extraneous modes, flexible structures, multi-input multi-output (MIMO) systems, stochastic system identification, tower cranes.

\section{Introduction}

Many mechanical and structural systems respond dynamically to random environmental loads, such as wind, wave or earthquake forces. Examples include flexible buildings vibrating due to turbulent wind loading and offshore structures moving as a result of combining wave and wind loading. To assess the reliability of such structures it is important to predict their dynamic response, at the design stage. However, whilst mass and stiffness parameters in the governing equations of motion can usually be computed with some accuracy, damping parameters are normally not quantifiable by theoretical means.

Vibration analyses of gantry crane system under swinging load, was studied by [1]. A mathematical model of the system dynamics are derived and numerically solved using finite element method. The study showed that the flexibility of the crane framework has a significant effect on the system dynamics.

Modeling and parameter identifications of crane vibrating systems are essentially needed in the design process of their controllers for both position and swing vibration. Since the identification process of crane systems is, troublesome and time consuming task, a practical and intelligent control method for automatic gantry crane is introduced by [2]. Their fuzzy logic control method is found to be practically applicable without the need to have the exact crane model.

To identify the parameters of these systems, their order, which roughly can be considered as the number of these parameters, must be known, and the system must be also represented in a canonical form, [311]. Otherwise, biased estimates of the parameters could be obtained, [3, 4, 7, 8]. Recently, several time domain identification techniques have been proposed, [3-8]. Identification accuracy is improved by increasing the model order. However, these over-specified models contain extraneous modes beside system modes. To distinguish between system modes and these extraneous modes, several techniques have been proposed, for single-input single output cases, [4-6]. However, the extraneous modes cannot be distinguished from system modes, unless physical restrictions are applied to the model.

In this work, a canonical form, that presents the structural dynamics, has been proposed and its uniqueness has been proved. The backwards approach has been applied to identify the system parameters, using an over-specified model. Regarding the order identification of MIMO systems, the pole-zero cancellation technique has been proposed. This proposed technique is capable of identifying both the order and parameters, using the minimum prediction error method. Several simulation examples are studied and presented here to illustrate the effectiveness of the proposed technique. Thereafter experimental runs were carried on a flexible truss system, the data of which has been used to identify the system parameters.

The present paper is organized as follows. In the following section, the problem formulation, together with backward methods and order estimation, has been introduced. The order identification technique, for the considered canonical form, is proposed afterward. The importance of over-specified model is illustrated through several simulation examples. In the next section, the experimental data from a flexible truss, presenting an 
overhead tower, is used to illustrate the effectiveness of the proposed approach. The conclusions are summarized in the last section

\section{Problem Formulation}

form:

The basic single-input single-output (SISO) discrete time-series model of a dynamic system has the

$$
y(k)=-a_{1} y(k-1)-a_{2} y(k-2)-\ldots-a_{n} y(k-n)+b_{0} u(k)+b_{1} u(k-1)+\ldots+b_{n} u(k-n)
$$

where $\mathrm{y}(\mathrm{k})$ is the system response at the kth discrete time, $\mathrm{u}(\mathrm{k})$ is the system input at the $\mathrm{k}^{\text {th }}$ discrete time and " $n$ " is the model order. The a's and b's are the model's parameters. This model is widely used to describe the dynamics of any finite-order linear system, [7]. The above representation is known as the forward form. Regarding the structural systems, the system response may be displacement, velocity or acceleration at any measuring point on the structure. Using z-transform, (1) can be written as;

$$
T_{F}(z)=\frac{b_{o} z^{n}+b_{1} z^{n-1}+\ldots .+b_{n}}{z^{n}+a_{1} z^{n-1}+\ldots .+a_{n}}
$$

The denominator polynomial is the model characteristic equation, and its roots can be used to estimate the natural frequencies and damping factors of the structure. The problem is to estimate the unknown model order according to which, the model parameters can be estimated. Unfortunately, it was noted that wrong estimates of the order leads to wrong estimates of the parameters, [3-8]. Several investigators have proposed efficient techniques to identify the order and parameters for SISO systems, [4-6].

In general, these techniques assume that in most cases the model is of a sufficient structure or it is an over-specified model. This simply means that the model order, is larger than or equal to the system order. In another work, [8], an author of this work proposed a solution for the order and parameter identification problem, using the forward method. However, this technique is efficient if the order of one of the elements in every row is known. Otherwise, it is difficult to distinguish between system and computational modes.

In this work, the backward model will be implemented to overcome the above difficulty. (1) can be written in the backward time-series model as follows:

$$
y(k)=-g_{1} y(k-1)-g_{2} y(k-2)-\cdots-g_{n} y(k-n)+d_{0} u(k)+d_{1} u(k-1)+\ldots+d_{n} u(k-n)
$$

The characteristic equation of the model is the same as that of the forward one, but the number of equations which are used for identification are effectively increased, [9]. Regarding (MIMO) systems, the problem is quite difficult. Considering the following MIMO system;

$$
A(z) Y(k)=G(z) U(k)+B(z) W(k)
$$

Where $\mathrm{Y}(\mathrm{k})$ is an $\mathrm{m}$-dimensional vector represents the system output; $\mathrm{U}(\mathrm{k})$ is an $\mathrm{p}$-dimensional vector represents the input of the system; and $\mathrm{W}(\mathrm{k})$ is an m-dimensional zero-mean stochastic white noise with positive definite covariance matrix. The elements of the matrices $A(z), G(z)$ and $B(z)$ are polynomials of "z". It is therefore, colored noise is considered. The parameters and order of this system are unknown. The problem is thus to identify both the order and parameters and to verify this identification.

The matrix equation, equation (4), which represents MIMO dynamic model, must uniquely represents the system, or in other words, the system must be in Canonical form. The conditions, under which the order and parameters can be identified, must be also obtained.

In the following section, a new Canonical form will be proposed, and its uniqueness will be proved. This new Canonical form will simplify the order identification problem. This is achieved by decoupling the identification of the whole system parameters into the identification of several smaller subsystems. An identification technique is also proposed to achieve the consistent estimates of the model order and parameters. Several examples are included to investigate the effectiveness of the proposed technique. 


\section{Order Identification Technique}

This section is divided into three subsections. In the first one, the present proposed Canonical form is introduced and its uniqueness has been proved. The identification technique for both the model order and parameters is introduced in the second subsection. Several simulation examples are included in the third subsection to illustrate the effectiveness of the proposed technique.

\subsection{The present proposed Canonical form}

Before proceeding with the uniqueness proof, the following conditions shall be considered;

1. The system matrices A (z), G (z) and B (z) are relatively left prime, or in other words the Smith form of $[A(z): G(z): B(z)]$ is $[I: 0: 0]$ is considered. This consideration means that the every state of the system, (3), is controllable either from $\mathrm{U}(\mathrm{k})$ or from $\mathrm{W}(\mathrm{k})$.

2. The inputs $\mathrm{U}(\mathrm{k})$ and $\mathrm{W}(\mathrm{k})$ are independent.

3. The matrix A ( $\mathrm{z}$ ) of the system, (3), will be assumed to be diagonal.

4. To insure that the process $Y(k)$ is stationary and invertible, all the zeroes of the determinants of $A(z)$ and $\mathrm{B}(\mathrm{z})$ lie outside the unit circle.

The representation of $Y(k)$ by $(3)$ is ensured to be unique considering that:

1. The system (4), is characterized by the matrices A (z), G (z) and B (z) with A (z) being diagonal.

2. The greatest common left divisor, GCLD, of $A(z), G(z)$ and $B(z)$ is a uni-modular matrix (condition 1 presented above). Proof: See Appendix A.

\subsection{Present identification scheme}

The least prediction error method will be implemented to identify the system order and parameters. Sufficient conditions, on the model structure, will be obtained to ensure consistent estimation of the parameters. Also, it will be shown that the proposed Canonical form simplifies the identification problem.

Provided that the model is of sufficient structure, the diagonality of A (z) makes it simple to obtain the greatest common left divisor, GCLD, of the estimated matrices $\hat{A}(z), \hat{G}(z)$, and $\hat{B}(z)$. This is attained by using the simple methods of finding the common factor between the diagonal element of $\hat{A}(z)$ and the elements of the corresponding rows of $\hat{G}(z)$ and $\hat{B}(z)$. The order is then identified after factoring this GCLD.

\subsubsection{Parameters Identification}

The unknown parameters of A (z), G (z) and B (z) can be estimated by minimizing the following prediction error loss function;

$$
P(N, \lambda)=\frac{1}{N} \sum_{k=0}^{N-1} \xi^{T}(N, \lambda) \xi(N, \lambda)
$$

Where $\mathrm{N}$ is the number of measured data, $\mathrm{P}$ is the loss function and the prediction error $\xi(N, \lambda)$ is obtained from the following model, [10];

$$
\hat{A}(z) Y(k)=\hat{G}(z) U(k)+\hat{B}(z) \xi(k, \lambda)
$$

$\lambda$ is a vector that made up of all the unknown coefficients of the model matrices, $\hat{A}(z), \hat{G}(z)$ and

$\hat{B}(z)$. Under the ergodicity assumption, it can be shown that the loss function, equation (5), converges uniformly in $\lambda$, as the number of measured data $\mathrm{N}$ increases, to a deterministic function, $\mathrm{P}(\lambda)$, with a probability of one, [10]. This function, $\mathrm{P}(\lambda)$, is the global minimum of the loss function $\mathrm{P}(\mathrm{N}, \lambda),[10]$. The above result will be used to investigate the properties of the identified matrices $\hat{A}(z), \hat{G}(z)$ and $\hat{B}(z)$ at this global minimum as follows:

Considering the system, (4), and the above model, (5) and supposing that $\mathrm{U}(\mathrm{k})$ is persistently exciting of order "L", where "L" is the order of the matrix $\left[\hat{B}^{-1} \hat{A}\left(A^{-1} G-\hat{A}^{-1} \hat{G}\right)\right]$, and the model is of sufficient structure, the global minimum of the asymptotic loss function $\mathrm{P}(\lambda)$ will be corresponding to;

$$
\begin{aligned}
& \hat{\boldsymbol{A}}(z)=\boldsymbol{R}(z) \boldsymbol{A}(z) \quad \hat{\boldsymbol{G}}(z)=\boldsymbol{R}(z) \boldsymbol{G}(z) \quad \hat{\boldsymbol{B}}(z)=\boldsymbol{R}(z) \boldsymbol{B}(z) \\
& \text { Where R(z) is a diagonal matrix representing the GCLD between the matrices }
\end{aligned}
$$




\subsubsection{System Modes and Extraneous Modes}

The properties of the system modes and extraneous modes will be discussed. For backward method, the system poles are outside the unit circle. If the data is noise-free, the minimum norm solution will imply a minimum phase system, and the computational or extraneous poles will fall inside the unit circle, [11]. Regarding the present case, it worth noting that with stochastic systems of colored noise, the computational poles are sometimes found outside the unit circle.

\subsection{Simulation Examples}

In what follows, several simulation examples will be represented to illustrate the present identification scheme. The input $\mathrm{U}(\mathrm{k})$ is considered zero, while the noise sequence $\mathrm{W}(\mathrm{k})$ is taken as a sample of a Gaussian random vectors of zero mean and unity variance. Therefore, the system, (4) can be written as;

$A(z) Y(k)=B(z) W(k)$

while the model equation can be written as follows;

$$
\hat{A}(z) Y(k)=\hat{B}(z) \xi(k, \lambda)
$$

The present identification scheme has been applied to the generated set of data. Three different cases have been considered. In the first case the model was an over-specified model. In the second case, the model order was taken equal to the system order. The model in the third case was not of sufficient structure, and wrong estimates of the parameters had been obtained.

3.3.1 Case 1, over-specified model

In this case, the system matrices are considered as follows;

$$
A(z)=\left[\begin{array}{cc}
1+0.8 z & 0 \\
0 & 1+0.55 z
\end{array}\right] \quad, \quad B(z)=\left[\begin{array}{cc}
1+0.37 z & 0.25 z \\
0.25 z & 1+0.8 z
\end{array}\right]
$$

The matrices of the identified model are taken as;

$$
\begin{aligned}
& \hat{A}(z)=\left[\begin{array}{cc}
1+a_{11} z+a a_{11} z^{2} & 0 \\
0 & 1+a_{22} z+a a_{22} z^{2}
\end{array}\right] \\
& \hat{B}(z)=\left[\begin{array}{cc}
1+b_{11} z+b b_{11} z^{2} & b_{12} z+b b_{12} z^{2} \\
b_{21} z+b b_{21} z^{2} & 1+b_{22} z+b b_{22} z^{2}
\end{array}\right]
\end{aligned}
$$

The estimated values of the above matrices are considered as;

$$
\begin{aligned}
& \hat{A}(z)=\left[\begin{array}{cc}
1-1.196 z-1.61 z^{2} & 0 \\
0 & 1+4.061 z-2.44 z^{2}
\end{array}\right] \\
& \hat{B}(z)=\left[\begin{array}{cc}
1-1.606 z-0.82 z^{2} & 0.248 z-0.47 z^{2} \\
0.236 z-1.06 z^{2} & 1-3.683 z-4.04 z^{2}
\end{array}\right]
\end{aligned}
$$

These matrices can be written according to equation (7) as;

$$
\begin{aligned}
& \hat{A}(z)=\left[\begin{array}{cc}
1-2 z & 0 \\
0 & 1-4.5 z
\end{array}\right]\left[\begin{array}{cc}
1+0.8 z & 0 \\
0 & 1+0.542 z
\end{array}\right] \\
& \hat{B}(z)=\left[\begin{array}{cc}
1-2 z & 0 \\
0 & 1-4.5 z
\end{array}\right]\left[\begin{array}{cc}
1+0.394 z & 0.248 z \\
0.236 z & 1+0.816 z
\end{array}\right]
\end{aligned}
$$

Therefore, the GCLD, R (z), between these identified matrices can be written as;

$$
R(z)=\left[\begin{array}{cc}
1-2 z & 0 \\
0 & 1-4.5 z
\end{array}\right]
$$

System matrices, $\bar{A}(z)$ and $\bar{B}(z)$, can be estimated as;

$$
\bar{A}(z)=\left[\begin{array}{cc}
1+0.8 z & 0 \\
0 & 1+0.542 z
\end{array}\right] \quad, \quad \bar{B}(z)=\left[\begin{array}{cc}
1+0.394 z & 0.248 z \\
0.236 z & 1+0.816 z
\end{array}\right]
$$

Which are a good estimate of the system matrices, $\mathrm{A}(\mathrm{z})$ and $\mathrm{B}(\mathrm{z})$.

\subsubsection{Case 2, model order is almost known}

Sometimes, the number of the parameters of an element of the system matrices is known beforehand. Hence, there is common factor to be sorted at the row of this element. In the present case, the system matrices will be considered as follows; 


$$
A(z)=\left[\begin{array}{cc}
1+0.8 z & 0 \\
0 & 1+0.55 z
\end{array}\right] \quad, \quad B(z)=\left[\begin{array}{cc}
1+0.35 z & 0 \\
0 & 1+0.85 z
\end{array}\right]
$$

The number of the system parameters of the matrix will be considered to be known beforehand, and hence the matrices of the identified model are taken as;

$$
\hat{A}(z)=\left[\begin{array}{cc}
1+a_{11} z+a a_{11} z^{2} & 0 \\
0 & 1+a_{22} z+a a_{22} z^{2}
\end{array}\right] \quad, \quad \hat{B}(z)=\left[\begin{array}{cc}
1+b_{11} z & b_{12} z \\
b_{21} z & 1+b_{22} z
\end{array}\right]
$$

and the estimate of the system matrices, $\bar{A}(z), \bar{B}(z)$, can be written as;

$$
\bar{A}(z)=\left[\begin{array}{cc}
1+0.76 z-0.02 z^{2} & 0 \\
0 & 1+0.58 z+0.03 z^{2}
\end{array}\right] \quad, \quad \bar{B}(z)=\left[\begin{array}{cc}
1+0.33 z & 0 \\
0 & 1+0.86 z
\end{array}\right]
$$

The extra elements are almost zero, and the estimates are in good agreement with the system matrices.

\subsubsection{Case 3, model order is not sufficient}

In this case the number of model parameters is less than that of the system. This will lead to wrong estimates of the parameters. The system matrices will be considered as follows;

$$
A(z)=\left[\begin{array}{cc}
1+0.8 z & 0 \\
0 & 1+0.75 z
\end{array}\right] \quad, \quad B(z)=\left[\begin{array}{cc}
1+0.35 z & 0.25 z \\
0.25 z & 1+0.75 z
\end{array}\right]
$$

The matrices of the identified model are taken as;

$$
\hat{A}(z)=\left[\begin{array}{cc}
1+a_{11} z & 0 \\
0 & 1
\end{array}\right] \quad, \quad \hat{B}(z)=\left[\begin{array}{cc}
1+b_{11} z & b_{12} z \\
b_{21} z & 1+b_{22} z
\end{array}\right]
$$

The estimated values of the above matrices are written as;

$$
\bar{A}(z)=\left[\begin{array}{cc}
1+0.73 z & 0 \\
0 & 1
\end{array}\right] \quad, \quad \bar{B}(z)=\left[\begin{array}{cc}
1+0.33 z & 0.24 z \\
0.24 z & 1-0.04 z
\end{array}\right]
$$

Wrong estimates have been obtained, and this illustrates that the system must be of sufficient structure.

\section{Experimental verification of the present identification technique}

Experimental data is used to verify the effectiveness of the present identification approach. A test rig was set up to experimentally investigate the vibration of a flexible structure representing the tower of an overhead tower cranes. An aluminum truss $12 \mathrm{~m}$ long and $0.55 \mathrm{~m}$ square cross section, the layout of which is shown in Fig. 1, was constructed.

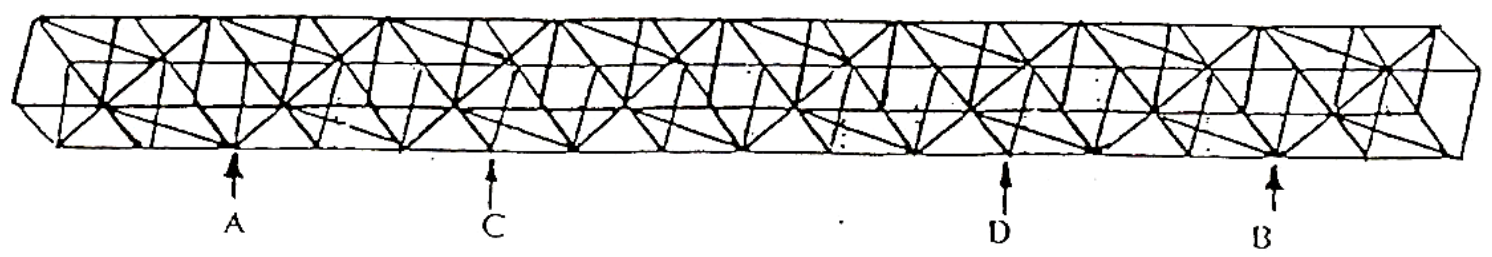

Figure1 Layout of the present vibrating structure

The truss is vertically flexibly fixed at its end near point $\mathrm{B}$. It is disturbed using two shakers of $1 \mathrm{~N}$ and $20 \mathrm{~N}$ forces at points A and B, $1.5 \mathrm{~m}$ apart of each truss end, respectively. A stochastic loading condition is applied through driving the two shakers by random wave generators. The velocities at points $\mathrm{A}$ and $\mathrm{B}$ are considered as inputs to the vibrating system and velocities at points $\mathrm{D}$ and $\mathrm{C}, 3.5 \mathrm{~m}$ apart of the truss center at each side, are considered as the system response. Velocities have been recorded using piezo-electric accelerometers with charge amplifiers that are provided with active integrating circuits to measure the velocity at the specified points. The data are collected at a sampling rate of $130 \mathrm{~Hz}$, and transferred to be processed by a personal computer through data acquisition system.

Fig. 2 and Fig. 3 illustrate a sample of the velocities at points B, input signal, and C, system response, respectively. 


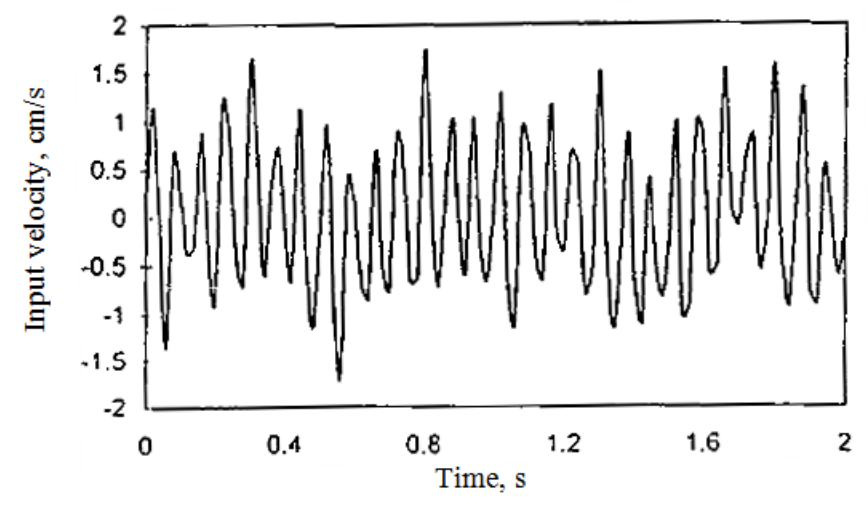

Figure 2 Sample of velocity input signal measured at point B

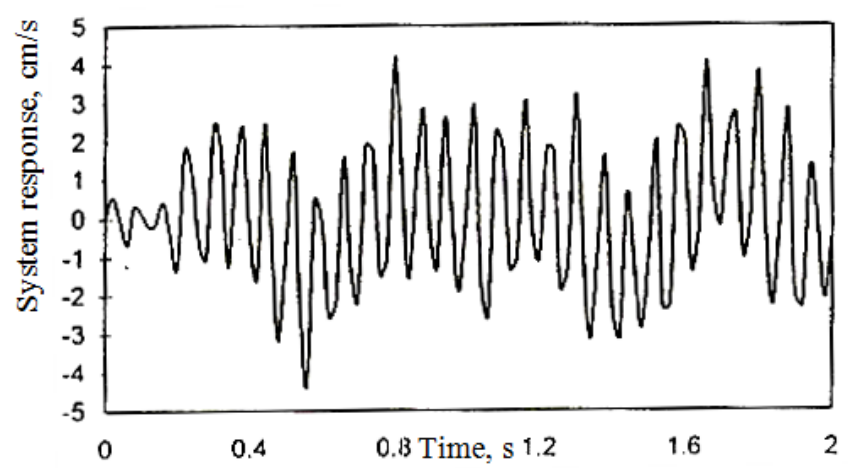

Figure 3 Sample of system velocity response measured at point $C$

A computer soft ware program was developed to apply the proposed identification technique to estimate the order and the parameters of the vibrating truss shown in figure 1. The program utilizes the Powell's minimization method and considers the minimum error as the norm of the errors at points $\mathrm{C}$ and $\mathrm{D}$. The program starts with a model order of four and increases the order by a step of two. The results of the present identification scheme are illustrated in Table 1 below.

Table 1: The results of the present identification scheme

\begin{tabular}{|c|l|l|l|}
\hline $\begin{array}{c}\text { Model } \\
\text { Order }\end{array}$ & $\begin{array}{c}\text { Minimum } \\
\text { Error }\end{array}$ & Identified Frequencies & \multicolumn{1}{|c|}{$\begin{array}{c}\text { Common Frequency } \\
\text { GCLD }\end{array}$} \\
\hline 10 & 0.2 & $* * * * *$ & $* * * * *$ \\
\hline 12 & 0.05 & $88,175,250 \mathrm{rad} / \mathrm{s}$ & $* * * * *$ \\
\hline 40 & 0.049 & $88,175,250 \mathrm{rad} / \mathrm{s}$ & $55 \mathrm{rad} / \mathrm{s}$ \\
\hline 50 & 0.0485 & $88,175,250 \mathrm{rad} / \mathrm{s}$ & $55,150 \mathrm{rad} / \mathrm{s}$ \\
\hline 80 & 0.032 & $88,175,250,8,14 \mathrm{rad} / \mathrm{s}$ & $150,230,200 \mathrm{rad} / \mathrm{s}$ \\
\hline
\end{tabular}

The above results illustrate that the system frequencies cannot be identified if the model order is less than twelve. The minimum error also is a sensitive indicator to the system frequencies. For example, it has been decreased to $25 \%$ of its value when identifying the highest natural frequencies, 88,175 and $250 \mathrm{rad} / \mathrm{s}$. On the other hand, negligible changes in the minimum error have been noticed when identifying the common frequencies, as they are canceled by the identified numerator part according to the pole-zero cancellation technique.

However, some of the extraneous poles lie outside the unit circle. This can attributed to the noise associated with the measured input data. The results of, [11] show that the extraneous poles lie inside the unit circle if the data is noise free.

The experimental velocity data at points A and B are used as online inputs to the identified model derived for the present flexible truss. The system responses at points $\mathrm{C}$ and $\mathrm{D}$ are calculated by the model and compared to the corresponding measured results. This is done to verify the effectiveness of the present model. The verification of velocities data from 2 - 3 seconds is illustrated in Fig. 4. The figure shows both the calculated and experimental data plotted on the $\mathrm{X}$ and $\mathrm{Y}$ axes respectively. It is clear that the dispersion of the best fit line of the data from the $45^{\circ}$ line, equal data values of both axes, is very small. This verifies the effectiveness of the present identified model. 


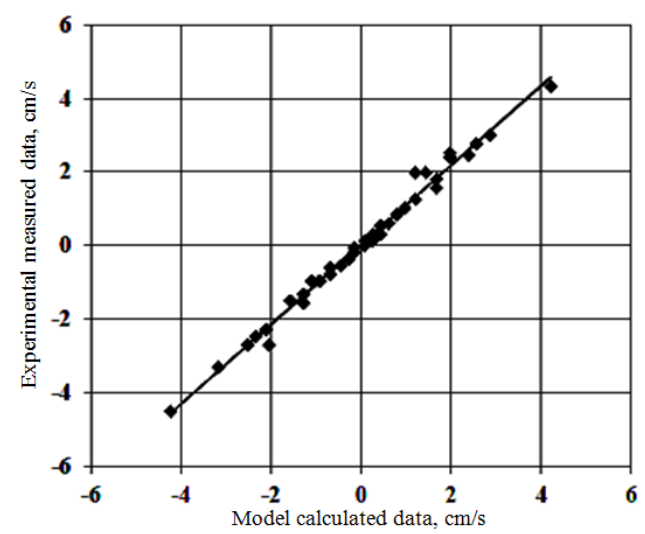

Fig. 4 Verification of the identified model, from $t=2-3 \mathrm{sec}$.

\section{Conclusions}

The following conclusions may be drawn regarding the present identification technique for MIMO systems and the proposed canonical form.

1. The implementation of the proposed canonical form simplifies the identification problem by decoupling the identification of the whole system into the identification of several smaller subsystems.

2. The increase of the model order produces extra identified modes, often associated with a decrease of the minimum prediction error.

3. The simulation examples illustrate the effectiveness of the proposed method. It is found that the extraneous modes can be distinguished from the system modes by the factorization method.

4. The extraneous modes, generally, lie inside the unit circle.

5. The results of the identified model have been verified using actual experimental measured data for a flexible structure. The extra modes have been identified, and a small dispersion, from the actual data, has been found by the verification.

\section{Appendix A}

In what follows, it will be proved that the model, proposed in section 3.1 of this work, uniquely represents the system. The proof will carried out using the contradiction concept.

Assume that the matrices $A(z), G(z)$ and $B(z)$ and $A_{0}(z), G_{0}(z)$ and $B_{0}(z)$ are two representations for the considered system, with $A(z)$ and being diagonal. Denote the GCLD of $[A(z), G(z)$ and $B(z)]$ and $\left[A_{0}(z)\right.$, $\mathrm{G}_{\mathrm{o}}(\mathrm{z})$ and $\left.\mathrm{B}_{\mathrm{o}}(\mathrm{z})\right]$ by the unimodular matrices, $\mathrm{P}$ and $\mathrm{P}_{\mathrm{o}}$, respectively. It can be proved that there exist two diagonal matrices $\mathrm{H}$ and $\mathrm{K}$ such that;
$\mathbf{H A}=\mathbf{K} \mathbf{A}_{\mathbf{0}}$
$\mathbf{H} \mathbf{B}=\mathbf{K} \mathbf{B}_{0}$
$\mathbf{H ~ G}=\mathbf{K ~ G}_{\mathbf{0}}$

Since $P$ and $P_{o}$, the uni-modular of $[A(z), G(z)$ and $B(z)]$ and $\left[A_{o}(z), G_{o}(z)\right.$ and $\left.B_{o}(z)\right]$ respectively, are represented according to the Smith form [7], as;

$\mathrm{U}[\mathrm{A}(\mathrm{z}), \mathbf{G}(\mathrm{z})$ and $\mathrm{B}(\mathrm{z})] \quad \mathrm{V}=[\mathbf{I}, \mathbf{O}, \mathbf{O}], \quad \mathrm{U}_{\mathrm{o}}\left[\mathrm{A}_{\mathbf{0}}(\mathrm{z}), \mathbf{G}_{\mathbf{0}}(\mathrm{z})\right.$ and $\left.\mathrm{B}_{\mathbf{0}}(\mathrm{z})\right] \quad \mathrm{V}_{\mathbf{0}}=[\mathbf{I}, \mathbf{O}, \mathbf{O}] \quad$ (A.2)

Where $U$ and $U_{o}$ are square unimodular matrices of dimension $m$, and $V$ and $V_{o}$ are unimodular

matrices of dimension $(2 \mathrm{~m}+\mathrm{p})$. Now the above equations can be written as;

$$
\left[H U^{-1}, 0,0\right]\left[\begin{array}{ll}
V_{11} & V_{12} \\
V_{21} & V_{22}
\end{array}\right]=\left[K U_{o}^{-1}, 0,0\right]
$$

This gives the following result;

$$
\boldsymbol{H} \boldsymbol{U}^{-1} \boldsymbol{V}_{11}=\boldsymbol{K} \boldsymbol{U}_{\boldsymbol{o}}^{-1} \text { and } \boldsymbol{V}_{12}=\mathbf{0}
$$

Since $V_{11}$ and $V_{22}$ are uni-modular matrices, the above equation can be written as;

$$
\boldsymbol{H}=\boldsymbol{K} \boldsymbol{U}^{\boldsymbol{0}}
$$

Where, $\boldsymbol{U}^{\boldsymbol{0}}$ is a diagonal unimodular matrix

Because of that $\mathrm{H}$ and $\mathrm{K}$ are diagonal matrices and since $\mathrm{A}(0)=\mathrm{I}$, (A.1) gives the following result;

$$
\begin{aligned}
& \mathbf{H}=\mathbf{K} \text { and Therefore, } \quad \mathbf{A}=\mathbf{A}_{\mathbf{0}} \quad \mathbf{B}=\mathbf{B}_{\mathbf{o}} \quad \mathbf{G}=\mathbf{G}_{\mathbf{0}} \\
& \text { And thus the present Canonical form is a unique representation for the system. }
\end{aligned}
$$

\section{References}

[1] E. Yazid, S. Parman, and K. Fuad, Vibration Analysis of Flexible Gantry Crane System Subjected Swinging Motion of Payload, Journal of Applied Science, ISSN 1812-5654, 11 (10), 2011, 1701-1715.

[2] J. Wahyudi, M. Riza, and J. Momoh, , Control Strategy for Automatic Gantry Crane Systems: A Practical and Intelligent Approach, International Journal of Advanced Robotic Systems, ISSN 1729-8806, Vol. 4, No. 4, 2007, 447-456. 
[3] R. L. Kashyap, and R. E. Nasburg, Parameter estimation in multivariable stochastic difference equations, IEEE Trans., Vol.AC-19, 1974, 784-790.

[4] H Akiake, A new look at the statistical model identification, IEEE Trans., Vol.AC-19, 1974, $216-222$.

[5] J. R. Trapero, H. Sira-Ramírez, and V. F. Feliu Batlle, A fast on-line frequency estimator of lightly damped vibrations in flexible structures, J. of sound and Vibration Volume 307, Issues 1-2, 2007, 365-378.

[6] M. Vasta, and J. B. Roberts, Stochastic parameters estimation of non-linear systems using only higher order spectra of the measured response, Journal of Sound and Vibration, 213 (2), 1998, 201-221.

[7] P. Eykhoff, System Identification (John Wiley \& sons, London, 1974).

[8] T. G. Abu-El-Yazied, On the Modeling and Prediction of the Air Pollutants Stochastic Dispersion, 1st Jordanian Mech. Eng. Conf., Amman, Jordan, 1995, 638-645.

[9] Y. Rolain, J. Schoukens, and R. Pintelon, Order Estimation for Linear Time-Invariant Systems using Frequency Domain Identification Methods, IEEE Trans., Vol.AC-42, 1997, 1408-1417.

[10] J. B. Roberts, and M. Vasta, Energy-Based Stochastic Estimation for Nonlinear Oscillators with Random Excitation, Journal of Applied Mechanics, vol. 67(4), 2000, 763-771.

[11] K. J. Keesman, Weighted Least Squares Set Estimation from 1 to $\infty$ Norm Bounded Noise Data, IEEE Trans., Vol.AC-42, 1997, 1456-1459.
Abbreviations
SISO single-input single-output
MIMO multi-inputs multi-outputs
GCLD greatest common left divisor 